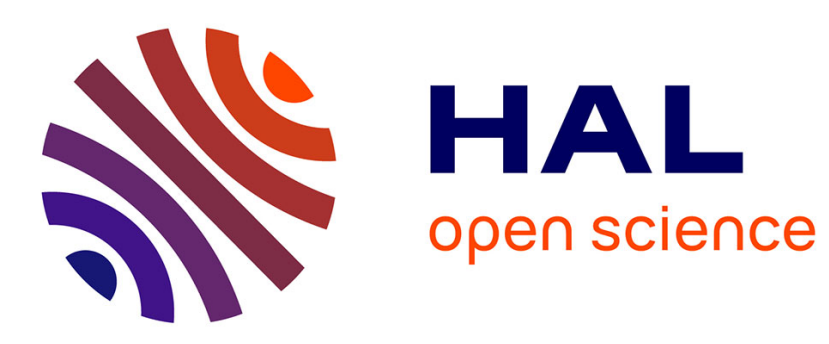

\title{
New routes to diethyl \\ 1-fluoromethylphosphonocarboxylates and diethyl 1-fluoromethylphosphonocarboxylic acid
}

\author{
Rachel Waschbüsch, John Carran, Philippe Savignac
}

\section{- To cite this version:}

Rachel Waschbüsch, John Carran, Philippe Savignac. New routes to diethyl 1fluoromethylphosphonocarboxylates and diethyl 1-fluoromethylphosphonocarboxylic acid. Tetrahedron, 1997, 53 (18), pp.6391-6400. 10.1016/S0040-4020(97)00312-8 . hal-03166382

\section{HAL Id: hal-03166382 \\ https://hal.science/hal-03166382}

Submitted on 11 Mar 2021

HAL is a multi-disciplinary open access archive for the deposit and dissemination of scientific research documents, whether they are published or not. The documents may come from teaching and research institutions in France or abroad, or from public or private research centers.
L'archive ouverte pluridisciplinaire HAL, est destinée au dépôt et à la diffusion de documents scientifiques de niveau recherche, publiés ou non, émanant des établissements d'enseignement et de recherche français ou étrangers, des laboratoires publics ou privés. 


\title{
New Routes to Diethyl 1-Fluoromethylphosphonocarboxylates and Diethyl 1-Fluoromethylphosphonocarboxylic Acid
}

\author{
Rachel Waschbüsch, John Carran and Philippe Savignac \\ Laboratoire Hétéroéléments et Coordination, URA CNRS 1499, DCPH, Ecole Polytechnique \\ 91128 Palaiseau Cedex, France. ${ }^{\text {a }}$ Tel. (+33) 01693345 79. Fax. (+33) 0169333990.
}

\begin{abstract}
Diethyl 1-fluoromethylphosphonocarboxylic esters 4, bearing a variety of ester groups (alkyl, aryl), have been conveniently prepared in a one-pot process, by reaction of chloroformates with 1-lithio1-fluoro-1-(trimethylsilyl)methylphosphonate 2 derived from 1,1-dibromo-1-fluoromethylphosphonate 1. The overall yields generally range from 80 to $91 \%$. Reaction of 2 with $\mathrm{CO}_{2}$ leads to a novel efficient synthesis of diethyl 1-fluoromethylphosphonocarboxylic acid 7 in $86 \%$ yield.
\end{abstract}

\section{INTRODUCTION}

A variety of monofluoro compounds exhibit remarkable biological activities ${ }^{1}$ and the compounds are in ever increasing demand from medical and biological sciences. Biologically active molecules containing a vinylic fluorine atom are of special interest, ${ }^{2}$ as this moiety is present in a number of enzyme inhibitors. ${ }^{3}$ 1-Fluoromethylphosphonocarboxylic acid derivatives are currently being explored and evaluated as general precursors of $\alpha$-fluoro- $\alpha, \beta$-unsaturated esters by the Wittig-Horner reaction which provides an efficient and convenient synthesis of fluoroacrylates of defined geometry. ${ }^{4}$ The potential value of these fluoroacrylates in biology and as synthetic building blocks has stimulated much interest in their synthesis. During recent decades research into convenient and efficient methods for the development of syntheses of $\alpha$-fluoroacrylic acid derivatives has been systematically pursued with the aim of studying the properties and applications of fluoropolymers based on monomers of this class. ${ }^{5}$ It has now been well established that, in many respects, poly(methyl $\alpha$-fluoroacrylates) are considerably superior to poly(methyl methacrylate) as regards their physicomechanical properties. A large number of $\alpha$-fluoroacrylic acid derivatives (e.g. halides, amides, nitriles, alkyl and aryl esters) have been synthesized and the preparation of most of these derivatives has been carried out in order to obtain polymers with valuable properties. In this paper, we exploit earlier work from this laboratory which suggests that the 1-lithio-1-fluoro-1-(trimethylsilyl)methylphosphonate carbanion 2 , readily prepared by a double halogen-metal exchange in the presence of chlorotrimethylsilane from dibromofluoromethylphosphonate 1, should have great synthetic potential. 6 In view of this we decided to explore in detail the reaction between our carbanion 2 and several chloroformates $(\mathrm{Cl}-\mathrm{C}(\mathrm{O}) \mathrm{O}-\mathrm{R})$ in order to prepare a variety of 1-fluoromethylphosphonocarboxylates containing different ester groups. While there are a number of reports in the literature relating the synthesis of diethyl 1-fluoromethylphosphonocarboxylic methyl,

a E-mail: dcph@poly.polytechnique.fr 
ethyl or $t$-butyl esters, ${ }^{7}$ we are unaware of any reports relating to the synthesis of other diethyl 1-fluoromethylphosphonocarboxylic alkyl or aryl esters.

\section{RESULTS AND DISCUSSION}

The current synthetic approach to 1-fluoromethylphosphonocarboxylates exploits a thermal MichaelisArbuzov reaction employing methyl or ethyl 1-bromo-1-fluoroacetate and triethylphosphite (Scheme 1). ${ }^{2 a, 7 b, 8}$ However the alkyl 1-bromo-1-fluoroacetates are not trivial starting materials (several preparations of these compounds have been described in the literature $)^{8 \mathrm{~b}}$ and the yield of the Michaelis-Arbuzov reaction is at best $65-71 \%(\mathbf{4 a}, \mathbf{c})$. In addition, it is easy to modify the ester groups of the phosphite but any modification of the carboxylic ester group requires access to new 1-bromo-1-fluoroacetates.

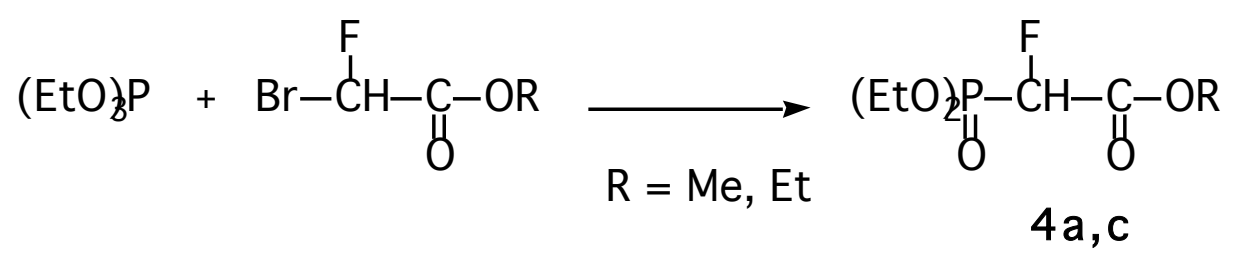

Scheme 1.

The Michaelis-Becker reaction between diethyl sodiophosphite and the methyl or ethyl 1-chloro1-fluoroacetates in benzene has also been described (Scheme 2)..$^{7 \mathrm{c}}$

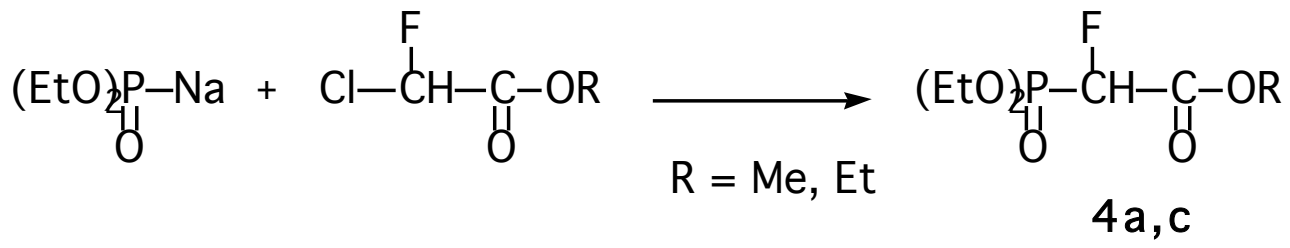

Scheme 2.

More recently the triethyl phosphonocarboxylate has been fluorinated in THF with N-fluoro$o$-benzenedisulfonimide (NFOBS) via the sodium enolate at $-78^{\circ} \mathrm{C}$ to give $4 \mathbf{c}$ in $78 \%$ yield (Scheme 3 ). $.7 \mathrm{~d}$

$$
\left(\mathrm{EtO}_{\underset{\mathrm{H}}{\mathrm{P}}-\mathrm{CH}_{2}-\mathrm{CH}-\mathrm{OEt}} \underset{\mathrm{NaHMDS}, \mathrm{THF}}{\stackrel{\mathrm{C}_{6} \mathrm{H}_{4}\left(\mathrm{SO}_{2}\right)_{2} \mathrm{NF}}{\mathrm{NaHMD}}}\right.
$$

Scheme 3.<smiles>CCOC(=O)C(F)[Po](=O)OCC</smiles>

$4 \mathrm{c}$

NFOBS, introduced by Davis and Han, is stable, easily prepared, and is the reagent of choice for the selective electrophilic monofluorination of enolates and carbanions (for a review of fluorination procedures see refs. $10 \&$ 11). However, as a consequence of the enhanced acidity of the $\alpha$-fluoro protons in the fluorocarboxylates, difluorination often competes with monofluorination. High temperatures and potassium enolates also promote difluorination (Scheme 4). 


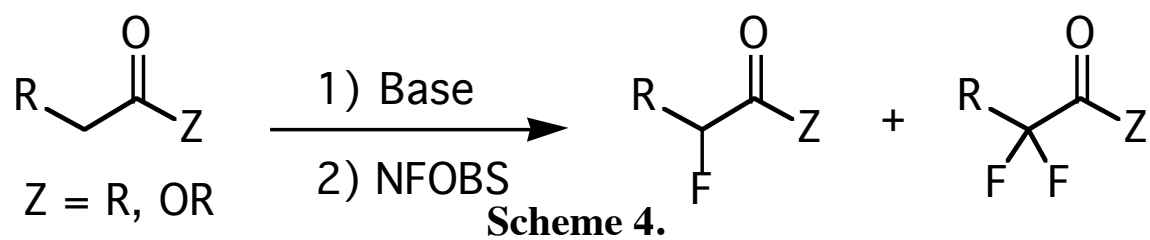

We have developed a much simpler and equally general procedure for obtaining 1-fluoromethylphosphonocarboxylates $\mathbf{4}$, in a one-pot sequence, by treating the 1-lithio-1-fluoro1-(trimethylsilyl)methylphosphonate $\mathbf{2}$ with readily available alkyl or aryl chloroformates $\mathrm{Cl}-\mathrm{C}(\mathrm{O}) \mathrm{O}-\mathrm{R}$ (Scheme 5).<smiles>CCOP(=O)(OCC)C(F)(Br)Br</smiles>

1<smiles>[R]OC(=O)C(F)[Pb](=O)OCC</smiles>

4

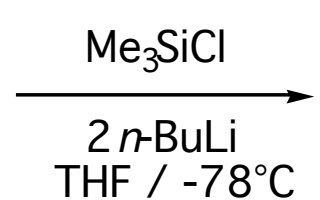

1) $\mathrm{EtOH}$

2) $2 \mathrm{M} \mathrm{HCl}$

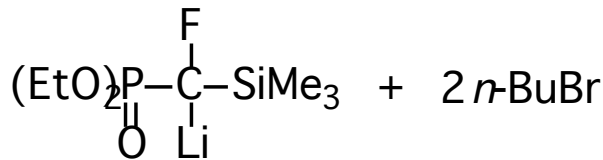

2<smiles>[R]OC(=O)Cl</smiles><smiles>[R]OC(=O)C(F)([Si])C(=O)OCC</smiles>

Scheme 5.

Preparative details, and spectroscopic evidence for the structure assignments for the carbanion $\mathbf{2}$ have been presented elsewhere. $6 \mathrm{~b}, 6 \mathrm{c}$ After formation at low temperature, $\mathbf{2}$ was reacted at the same temperature with freshly distilled chloroformates. We found that carbanion 2 reacted relatively slowly with chloroformates in THF at low temperature. Use of 1 eq. of chloroformate gave poor yields of adducts and substantial quantities of diethyl 1-fluoropentylphosphonate resulting from alkylation of 2 with butylbromide ( 2 eq.) generated in the reaction medium by the double halogen-metal exchange reaction. Under the latter conditions, formation of 1-fluoro-1-(trimethylsilyl)methylphosphonocarboxylates $\mathbf{3}$ competed to some extent with the direct alkylation of carbanion $\mathbf{2}$ by butylbromide. We discovered that carbanion $\mathbf{2}$ reacts with alkyl chloroformates much more readily than aryl chloroformates. Thus, to obtain complete conversion of carbanion $\mathbf{2}$ into 1-fluoro-1-(trimethylsilyl)methylphosphonocarboxylates $\mathbf{3}$, it is imperative to treat $\mathbf{2}$ with a large excess of chloroformate. Thus, 2 mol. eq. of alkyl chloroformates (primary or secondary) in $\mathrm{THF}$ at $-78^{\circ} \mathrm{C}$ for $30 \mathrm{~min}$ were necessary, and $2.5 \mathrm{~mol}$. eq. of vinyl or aryl chloroformates. The reaction failed in four cases, with trichloromethylchloroformate, trichloroethylchloroformate, $\alpha$-chloroallylchloroformate and adamantylchloroformate. The halogenated reagents were not compatible with the basic reaction conditions used, as a halogen-metal exchange reaction occurs and the formation of diethyl 1-chloro-1-fluoro1-(trimethylsilyl)methylphosphonate was observed as the major product (Scheme 6).<smiles>CCOC(=O)C(C)(F)Cl</smiles>

1) $\mathrm{ClCO}_{2}\left(\mathrm{CH}_{2}\right)_{n} \mathrm{CCl}_{3}$

2) $\mathrm{EtOH}$

3) $2 \mathrm{M} \mathrm{HCl}$ $\mathrm{n}=0,1$<smiles>CCOP(=O)(OCC)C(F)(Cl)SC</smiles> 


\section{Scheme 6.}

All these intermediates 3 were obtained as single products and assigned on the basis of their ${ }^{31} \mathrm{P}$ NMR spectra and of the observed coupling constants. Typically, the carbanion 2 displayed a signal as a doublet in the ${ }^{31} \mathrm{P}$ NMR at $\delta(\mathrm{THF})+52.8 \mathrm{ppm},{ }^{2} J_{\mathrm{PF}} 67.2 \mathrm{~Hz}$, whilst the signal of the intermediate 3 appeared at $\delta(\mathrm{THF})+14.2$ ppm, ${ }^{2} J_{\mathrm{PF}} 67.4 \mathrm{~Hz}$.

Compounds 3 were not isolated but converted directly in THF solution into 1-fluoromethylphosphonocarboxylates 4 . The conditions of removal of the TMS protecting group are not trivial and failure to follow the procedure herein resulted in low yields or destruction of the compounds. We have already described a procedure for the removal of the TMS group in 1-fluoro-1-(trimethylsilyl)methyl-phosphonates with EtOLi / EtOH.6c With the presence on the same carbon of a fluorine, phosphoryl and carboxylate groups, there was a serious risk that the product $\mathbf{3}$ would undergo fragmentation by nucleophilic attack at phosphorus. Indeed, under these conditions, our chloroformate adducts underwent cleavage of the C-P bond to give phosphates exclusively $\delta(\mathrm{THF}){ }^{31} \mathrm{P}-1.1 \mathrm{ppm}$. On the other hand, attempts to remove the TMS protecting group by direct acidic quenching of the THF solution with $5 \mathrm{M} \mathrm{HCl}$ at $0^{\circ} \mathrm{C}$ was no more effective and gave a mixture of byproducts containing silylated and non silylated moieties.

Table 1. 1-Fluoromethylphosphonocarboxylates 4

\begin{tabular}{|c|c|c|c|}
\hline Compound 4 & $\mathrm{R}$ & $\begin{array}{l}\text { chloroformate amount } \\
\text { (mol. eq.) }\end{array}$ & Yield (\%) \\
\hline $\mathbf{a}$ & $\mathrm{Me}$ & 2 & 91 \\
\hline $\mathbf{b}$ & $-\mathrm{CH}_{2} \mathrm{Cl}$ & 2 & 85 \\
\hline c & Et & 2 & 90 \\
\hline d & $-\mathrm{CH}_{2} \mathrm{CH}_{2} \mathrm{Cl}$ & 2 & 84 \\
\hline $\mathbf{e}$ & $n-\mathrm{C}_{8} \mathrm{~F}_{13} \mathrm{H}_{4}$ & 2 & 90 \\
\hline $\mathbf{f}$ & $-\mathrm{CH}_{2}-\mathrm{CH}=\mathrm{CH}_{2}$ & 2 & 87 \\
\hline g & $i-\mathrm{Bu}$ & 2 & 86 \\
\hline $\mathbf{h}$ & neo-pent & 2 & 87 \\
\hline $\mathbf{i}$ & $i$-Pr & 2 & 85 \\
\hline $\mathbf{j}$ & $-\mathrm{CH}\left(\mathrm{CH}_{2} \mathrm{Cl}\right)_{2}$ & 2 & 80 \\
\hline
\end{tabular}


$\mathbf{k}$

$-\mathrm{CH}=\mathrm{CH}_{2}$

I

$\mathrm{Ph}$

We now describe a much simpler and more effective procedure for the desilylation of the chloroformate analogues. This involved treating the intermediates 3 with anhydrous ethanol at $-78^{\circ} \mathrm{C}$ and subsequent warming to $0^{\circ} \mathrm{C}$ for $30 \mathrm{~min}$. In this way, after acidic quenching $(2 \mathrm{M} \mathrm{HCl})$ the desired 1-fluoromethylphosphonocarboxylates 4 were obtained and isolated after distillation as colorless liquids in very good overall yield (approx. 85\%) for the three steps starting from $\mathbf{1}$. The products 4 were found to be thermically stable and thus are easily purified by distillation at high temperatures (up to $200^{\circ} \mathrm{C}$ ) without decomposition.

In order to prepare the free 1-fluoromethyl(diethylphosphono)carboxylic acid 7 we attempted to hydrolyse the methyl ester $\mathbf{4 a}$ under acidic conditions $(5 \mathrm{M} \mathrm{HCl})$ rather than under basic conditions which generally results in fragmentation of products. Under our conditions only unchanged methyl ester 4a was recovered from the reaction mixture. By another route, we anticipated that treatment of the carbanion 2 with $\mathrm{CO}_{2}$, followed by acidic work up would provide access to 7 in a one-pot operation. Thus, a solution of the carbanion 2 in THF at $-60^{\circ} \mathrm{C}$ was treated with excess anhydrous $\mathrm{CO}_{2}$ until the exothermic reaction ceased. The $\alpha$-silylated intermediate 5 undergoes a [1,3]-migration on warming to give the silyloxy enolate 6 and then hydrolysis of the reaction medium with $3 \mathrm{M} \mathrm{HCl}$ at $20^{\circ} \mathrm{C}$, gave 7 in high yield ( $86 \%$ overall) as a crystalline white solid after ethereal extraction (Scheme 7). This reaction has been proved to be a reliable, novel synthesis of the carboxylic acid 7 which is a valuable reagent for the preparation of $\alpha$-fluoro- $\alpha, \beta$-unsaturated acids $.^{9}, 10$

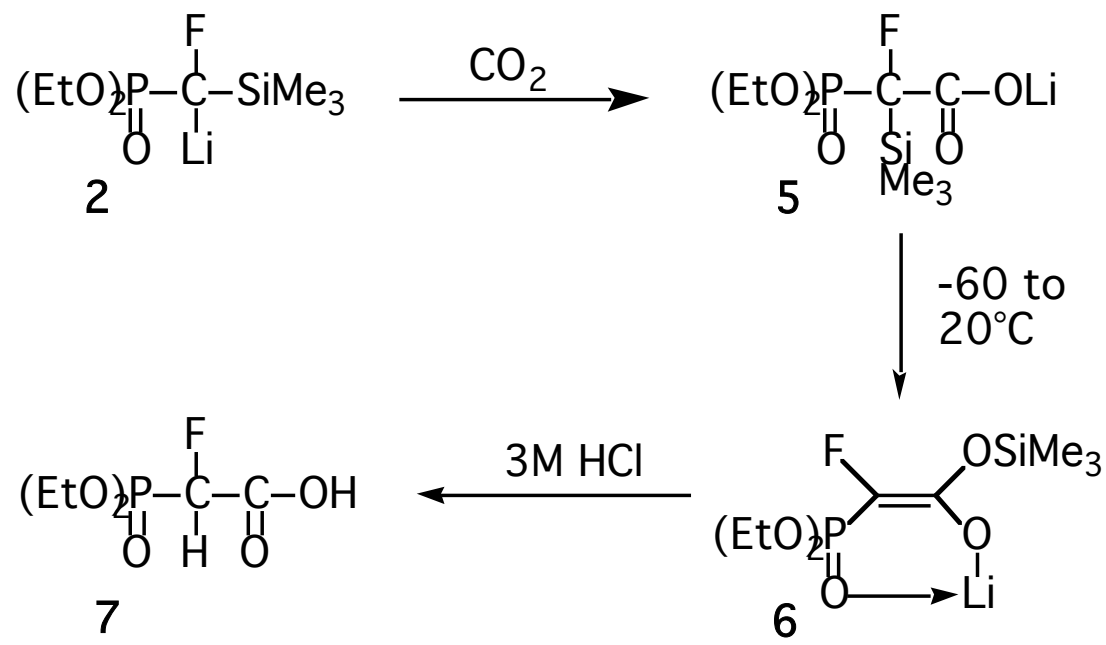

Scheme 7.

\section{CONCLUSION}

This article has further demonstrated the synthetic potential of the 1-lithio-1-fluoro1-(trimethylsilyl)methylphosphonate $\quad 2$. Novel 1-fluoromethyl-phosphonocarboxylates $\mathbf{4}$ have been 
synthesized. We believe that the results described provide the basis for a simple and efficient large-scale, onepot procedure for the high yield synthesis, starting from chloroformates, of 1-fluoromethylphosphonocarboxylates 4 which could be difficult to obtain by other means. It should find a wide application in their conversion into the corresponding fluoroacrylates. Additionally the new synthetic methodology described herein for the preparation of the free 1-fluoromethyl(diethylphosphono)carboxylic acid 7 will also be relevant and open new routes to $\alpha$-fluoro- $\alpha, \beta$-unsaturated acids which are currently being evaluated.

\section{EXPERIMENTAL SECTION}

NMR spectra were recorded on a Bruker AC 200 spectrometer operating at $200 \mathrm{MHz}$ for proton, 50.3 $\mathrm{MHz}$ for carbon and $81.01 \mathrm{MHz}$ for phosphorus. ${ }^{31} \mathrm{P}$ downfield shifts $(\delta)$ are expressed with a positive sign, in ppm, relative to external $85 \% \mathrm{H}_{3} \mathrm{PO}_{4}$ in $\mathrm{H}_{2} \mathrm{O} .{ }^{1} \mathrm{H}$ and ${ }^{13} \mathrm{C}$ chemical shifts $(\delta)$ are reported in ppm relative to $\mathrm{CDCl}_{3}$ as internal standard. Coupling constants $(J)$ are given in $\mathrm{Hz}$. The following abbreviations are used: s, d, t, q, m for singlet, doublet, triplet, quadruplet and multiplet respectively. Organic solvents were purified by standard procedures. THF was distilled under an inert atmosphere from purple solutions of sodiumbenzophenone ketyl. The synthesis of all compounds were carried out under dry nitrogen.

Diethyl 1,1-dibromo-1-fluoromethylphosphonate (1). see references.6b, 6c

General procedure for the synthesis of diethyl alkyl or aryl 1-fluoromethylphosphonocarboxylate (4) : To a solution of $n$-BuLi $(6.6 \mathrm{~mL}$ of a $1.6 \mathrm{M}$ solution in hexane, $10.5 \mathrm{mmol})$ in THF $(10 \mathrm{~mL})$ cooled to $-78^{\circ} \mathrm{C}$ is added dropwise a mixture of diethyl 1,1-dibromo-1-fluoromethylphosphonate 1 (1.64 g, $5 \mathrm{mmol}$ ) and TMSCl $(0.54 \mathrm{~g}, 5 \mathrm{mmol})$ in THF $(10 \mathrm{~mL})$ via a dropping funnel maintaining the temperature at $-78^{\circ} \mathrm{C}$. The reaction mixture was stirred at this temperature for $5 \mathrm{~min}$ and then the chloroformate $(10 \mathrm{mmol})$ in THF $(10 \mathrm{~mL})$ was added dropwise. The reaction mixture was again left to stir at $-78^{\circ} \mathrm{C}$ for $30 \mathrm{~min}$ before hydrolysis with absolute ethanol $(10 \mathrm{~mL})$. The reaction mixture was allowed to warm to $0^{\circ} \mathrm{C}$ within $30 \mathrm{~min}$ and then poured into a beaker of $\mathrm{HCl}(20 \mathrm{~mL}$ of $2 \mathrm{M}$ solution), dichloromethane $(20 \mathrm{~mL})$ and ice. The phases were separated and the aqueous phase was extracted with dichloromethane $(3 \times 20 \mathrm{~mL})$. The combined organic phases were dried over $\mathrm{MgSO}_{4}$ filtered and evaporated to yield the crude product which on bulb-to-bulb distillation give the title product $\mathbf{4 a - 1}$ as colorless liquids.

Diethyl methyl 1-fluoromethylphosphonocarboxylate (4a) : $\mathrm{C}_{7} \mathrm{H}_{14} \mathrm{O}_{5} \mathrm{PF}(\mathrm{M}=228)$

NMR ${ }^{31} \mathbf{P}\left(\mathrm{CDCl}_{3}\right)+10.0\left(\mathrm{~d},{ }^{2} \mathrm{~J}_{\mathrm{PF}}=71.4\right)$. / ${ }^{\mathbf{1}} \mathbf{H}\left(\mathrm{CDCl}_{3}\right) 1.32\left(\mathrm{tm}, 6 \mathrm{H},{ }^{3} \mathrm{~J}_{\mathrm{HH}}=7.1, \mathrm{CH}_{3} \mathrm{CH}_{2} \mathrm{O}\right), 3.83(\mathrm{~s}, 3 \mathrm{H}$,

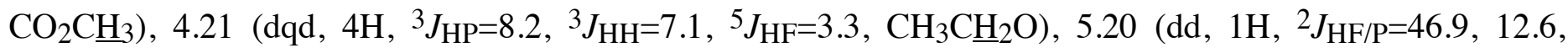

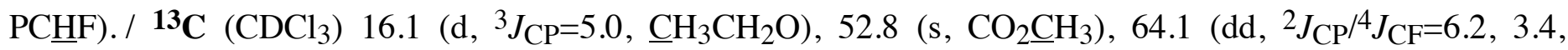
$\mathrm{CH}_{3} \underline{\mathrm{CH}}_{2} \mathrm{O}$ ), 84.8 (dd, ${ }^{1} J_{\mathrm{CF} / \mathrm{P}}=195.4,158.0, \mathrm{P} \underline{\mathrm{HFF}}$ ), $165.2\left(\mathrm{~d},{ }^{2} J_{\mathrm{CF}}\right.$ or $\left.\mathrm{CP}=22.0, \mathrm{PCHF}-\underline{C O}_{2}\right) . / \mathbf{~ m} / \mathbf{z}(\mathrm{EI}) 229$ $\left(\mathrm{M}^{+}+1,2 \%\right), 213(10), 197(8), 183(11)$.

Diethyl chloromethyl 1-fluoromethylphosphonocarboxylate (4b) : $\mathrm{C}_{7} \mathrm{H}_{13} \mathrm{O}_{5} \mathrm{PFCl}(\mathrm{M}=262.5)$

NMR ${ }^{31} \mathbf{P}\left(\mathrm{CDCl}_{3}\right)+8.3\left(\mathrm{~d},{ }^{2} \mathrm{~J}_{\mathrm{PF}}=70.5\right)$. / ${ }^{\mathbf{1}} \mathbf{H}\left(\mathrm{CDCl}_{3}\right) 1.36\left(\mathrm{tm}, 6 \mathrm{H},{ }^{3} \mathrm{~J}_{\mathrm{HH}}=7.1, \mathrm{C}_{3} \mathrm{CH}_{2} \mathrm{O}\right), 4.26(\mathrm{dqd}, 4 \mathrm{H}$,

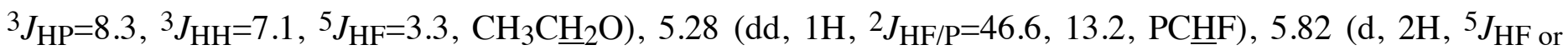


$\left.\mathrm{HP}=2.3, \mathrm{CO}_{2} \underline{\mathrm{C}}_{2}\right) . /{ }^{13} \mathrm{C}\left(\mathrm{CDCl}_{3}\right) 16.4\left(\mathrm{~d},{ }^{3} J_{\mathrm{CP}}=5.3 \underline{\mathrm{CH}}_{3} \mathrm{CH}_{2} \mathrm{O}\right), 64.7\left(\mathrm{~d},{ }^{2} J_{\mathrm{CP}}=6.5, \mathrm{CH}_{3} \underline{\mathrm{CH}_{2} \mathrm{O}}\right), 69.4(\mathrm{~s}$, $\left.\mathrm{CO}_{2} \underline{\mathrm{CH}}_{2}\right), 84.9$ (dd, ${ }^{1} J_{\mathrm{CF} / \mathrm{P}}=196.8,157.3, \mathrm{P} \underline{\mathrm{HF}}$ ), 163.5 (d, ${ }^{2} J_{\mathrm{CF}}$ or $\left.\mathrm{CP}=22.7, \mathrm{PCHF}-\underline{C O}_{2}\right) . / \mathrm{m} / \mathbf{z}(\mathrm{EI}) 263$ $\left(\mathrm{M}^{+}+1,{ }^{35} \mathrm{Cl}, 10 \%\right), 235\left({ }^{35} \mathrm{Cl}, 11\right), 207\left({ }^{35} \mathrm{Cl}, 22\right), 197(44)$.

Diethyl ethyl 1-fluoromethylphosphonocarboxylate (4c) : $\mathrm{C}_{8} \mathrm{H}_{16} \mathrm{FO}_{5} \mathrm{P}(\mathrm{M}=242)$

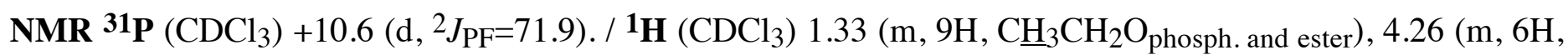

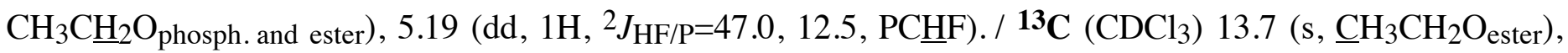
16.0 (dd, ${ }^{3} J_{\mathrm{CP}} / 5 J_{\mathrm{CF}}=5.8,4.5, \underline{\mathrm{CH}}_{3} \mathrm{CH}_{2} \mathrm{O}_{\text {phosph. }}$ ), 62.0 (s, $\mathrm{CH}_{3} \underline{\mathrm{CH}}_{2} \mathrm{O}_{\mathrm{ester}}$ ), 63.9 (dd, ${ }^{2} J_{\mathrm{CP}} /{ }^{4} J_{\mathrm{CF}}=5.4,2.7$, $\mathrm{CH}_{3} \underline{\mathrm{CH}}_{2} \mathrm{O}_{\text {phosph. }}$ ), 84.7 (dd, $\left.{ }^{1} J_{\mathrm{CF} / \mathrm{P}}=195.0,157.7, \mathrm{PCHF}\right), 164.5\left(\mathrm{~d},{ }^{2} J_{\mathrm{CF}}\right.$ or $\left.\mathrm{CP}=22.1, \mathrm{PCHF}-\mathrm{CO}_{2}\right) . / \mathbf{~ m} / \mathbf{z}(\mathrm{EI})$ $243\left(\mathrm{M}^{+}+1,8 \%\right), 214$ (18), 197 (39), 189 (53).

Diethyl 2-chloroethyl 1-fluoromethylphosphonocarboxylate (4d) : $\mathrm{C}_{8} \mathrm{H}_{15} \mathrm{FO}_{5} \mathrm{PCl}(\mathrm{M}=276.5)$

NMR ${ }^{31} \mathbf{P}\left(\mathrm{CDCl}_{3}\right)+9.6\left(\mathrm{~d},{ }^{2} J_{\mathrm{PF}}=71.9\right) . /{ }^{1} \mathbf{H}\left(\mathrm{CDCl}_{3}\right) 1.36\left(\mathrm{td}, 6 \mathrm{H},{ }^{3} J_{\mathrm{HH}}=7.1,{ }^{4} J_{\mathrm{HP}}=1.6, \mathrm{CH}_{3} \mathrm{CH}_{2} \mathrm{O}\right), 3.72(\mathrm{t}$, $\left.2 \mathrm{H},{ }^{3} J_{\mathrm{HH}}=5.7, \underline{\mathrm{H}}_{2} \mathrm{Cl}\right), 4.25\left(\mathrm{dqd}, 4 \mathrm{H},{ }^{3} J_{\mathrm{HP}}=8.2,{ }^{3} J_{\mathrm{HH}}=7.1,5 J_{\mathrm{HF}}=2.5, \mathrm{CH}_{3} \underline{C H}_{2} \mathrm{O}\right), 4.51\left(\mathrm{t}, 2 \mathrm{H},{ }^{3} J_{\mathrm{HH}}=5.7\right.$,

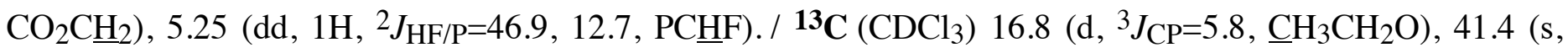
$\left.\underline{\mathrm{CH}}_{2} \mathrm{Cl}\right), 64.9\left(\mathrm{t},{ }^{2} J_{\mathrm{CP}}=5.3, \mathrm{CH}_{3} \underline{\mathrm{CH}}_{2} \mathrm{O}\right), 66.1\left(\mathrm{~s}, \mathrm{CO}_{2} \underline{\mathrm{CH}}_{2}\right), 85.4\left(\mathrm{dd},{ }^{1} J_{\mathrm{CF} / \mathrm{P}}=196.1,158.6, \mathrm{P} \underline{\mathrm{CHF}}\right), 165.1$ (d, ${ }^{2} J_{\mathrm{CF}}$ or $\left.\mathrm{CP}=22.1, \mathrm{PCHF}-\mathrm{CO}_{2}\right) . / \mathbf{m} / \mathbf{z}(\mathrm{EI}) 277\left(\mathrm{M}^{+}+1,{ }^{35} \mathrm{Cl}, 9 \%\right), 249\left({ }^{35} \mathrm{Cl}, 24\right), 217\left({ }^{35} \mathrm{Cl}, 41\right), 213(100)$.

Diethyl tridecafluorooctyl 1-fluoromethylphosphonocarboxylate (4e) : $\mathrm{C}_{14} \mathrm{H}_{15} \mathrm{O}_{5} \mathrm{PF}_{14}(\mathrm{M}=560)$

NMR 31P $\left(\mathrm{CDCl}_{3}\right)+9.5\left(\mathrm{~d},{ }^{2} J_{\mathrm{PF}}=71.9\right)$. / $\mathbf{1} \mathbf{H}\left(\mathrm{CDCl}_{3}\right) 1.36\left(\mathrm{t}, 6 \mathrm{H},{ }^{3} \mathrm{~J}_{\mathrm{HH}}=7.0, \mathrm{C}_{3} \mathrm{CH}_{2} \mathrm{O}\right), 2.95(\mathrm{tt}, 2 \mathrm{H}$, $\left.{ }^{3} J_{\mathrm{HF}}=18.2,{ }^{3} J_{\mathrm{HH}}=6.5, \mathrm{CO}_{2} \mathrm{CH}_{2} \mathrm{CH}_{2} \mathrm{CF}_{2}\right), 4.25\left(\mathrm{~m}, 4 \mathrm{H}, \mathrm{CH}_{3} \underline{\mathrm{CH}}_{2} \mathrm{O}\right), 4.57\left(\mathrm{t}, 2 \mathrm{H},{ }^{3} J_{\mathrm{HH}}=6.5, \mathrm{CO}_{2} \mathrm{CH}_{2} \mathrm{CH}_{2}\right)$, $5.23\left(\mathrm{dd}, 1 \mathrm{H},{ }^{2} J_{\mathrm{HF} / \mathrm{P}}=46.8,12.8, \mathrm{PC} \underline{\mathrm{HF}}\right) . /{ }^{13} \mathrm{C}\left(\mathrm{CDCl}_{3}\right) 16.7\left(\mathrm{~d},{ }^{3} J_{\mathrm{CP}}=5.8 \underline{\mathrm{CH}}_{3} \mathrm{CH}_{2} \mathrm{O}\right), 30.9\left(\mathrm{t},{ }^{2} J_{\mathrm{CF}}=21.8\right.$, $\left.\mathrm{CO}_{2} \mathrm{CH}_{2}-\mathrm{CH}_{2} \mathrm{CF}_{2}\right), 58.5\left(\mathrm{~s}, \mathrm{CO}_{2} \mathrm{CH}_{2}\right), 65.0\left(\mathrm{t},{ }^{2} J_{\mathrm{CP}}=6.2, \mathrm{CH}_{3} \underline{C H}_{2} \mathrm{O}\right), 85.5\left(\mathrm{dd},{ }^{1} J_{\mathrm{CF} / \mathrm{P}}=196.7,158.7, \mathrm{P} \underline{\mathrm{CHF}}\right)$, 104.1-126.4 (mt, $\left.{ }^{2} J_{\mathrm{CF}}=30.9-33.5,\left(\underline{\mathrm{CF}}_{2}\right)_{5} \underline{\mathrm{CF}}_{3}\right), 165.2\left(\mathrm{~d},{ }^{2} J_{\mathrm{CF} \text { or } \mathrm{CP}}=22.1, \mathrm{PCHF}-\underline{\mathrm{CO}}_{2}\right) . / \mathrm{m} / \mathbf{z}(\mathrm{EI}) 560\left(\mathrm{M}^{+}\right.$, $1 \%), 505(11), 485(7), 327(10)$.

Diethyl allyl 1-fluoromethylphosphonocarboxylate (4 f) : $\mathrm{C}_{9} \mathrm{H}_{16} \mathrm{O}_{5} \mathrm{PF}(\mathrm{M}=254)$

NMR ${ }^{31} \mathbf{P}\left(\mathrm{CDCl}_{3}\right)+10.0\left(\mathrm{~d},{ }^{2} \mathrm{~J}_{\mathrm{PF}}=71.9\right)$. / $\mathbf{1} \mathbf{H}\left(\mathrm{CDCl}_{3}\right) 1.34\left(\mathrm{tm}, 6 \mathrm{H},{ }^{3} \mathrm{~J}_{\mathrm{HH}}=7.1, \mathrm{CH}_{3} \mathrm{CH}_{2} \mathrm{O}\right), 4.33(\mathrm{dqd}, 4 \mathrm{H}$, $\left.{ }^{3} J_{\mathrm{HP}}=7.9,{ }^{3} J_{\mathrm{HH}}=7.1,5 J_{\mathrm{HF}}=3.2, \mathrm{CH}_{3} \mathrm{CH}_{2} \mathrm{O}\right), 4.74\left(\mathrm{dm}, 2 \mathrm{H},{ }^{3} J_{\mathrm{HH}}=5.8, \mathrm{CO}_{2} \mathrm{CH}_{2}\right), 5.21\left(\mathrm{dd}, 1 \mathrm{H},{ }^{2} J_{\mathrm{HF} / \mathrm{P}}=46.9\right.$, 12.7, PC타), $5.27\left(\mathrm{dm}, 1 \mathrm{H},{ }^{3} J_{\mathrm{HHcis}}=10.4, \mathrm{CO}_{2} \mathrm{CH}_{2}-\mathrm{CH}=\mathrm{CH}_{\mathrm{A}} \mathrm{H}_{\mathrm{B}}\right), 5.38\left(\mathrm{dm}, 1 \mathrm{H},{ }^{3} J_{\mathrm{HH} \text { trans }}=17.1, \mathrm{CO}_{2} \mathrm{CH}_{2}-\right.$ $\left.\mathrm{CH}=\mathrm{CH}_{\mathrm{A}} \underline{\mathrm{H}}_{\mathrm{B}}\right), 5.91\left(\mathrm{ddt}, 1 \mathrm{H},{ }^{3} J_{\mathrm{HHB}}=17.1,{ }^{3} J_{\mathrm{HHA}}=10.4,{ }^{3} J_{\mathrm{HH}}=5.8, \mathrm{CO}_{2} \mathrm{CH}_{2}-\mathrm{CH}=\mathrm{CH}_{\mathrm{A}} \mathrm{H}_{\mathrm{B}}\right) . /{ }^{13} \mathrm{C}\left(\mathrm{CDCl}_{3}\right)$ $16.7\left(\mathrm{~d},{ }^{3} J_{\mathrm{CP}}=5.0 \underline{\mathrm{CH}}_{3} \mathrm{CH}_{2} \mathrm{O}\right), 64.7\left(\mathrm{dd},{ }^{2} J_{\mathrm{CP}}=6.1,{ }^{4} J_{\mathrm{CF}}=2.9, \mathrm{CH}_{3} \underline{\mathrm{CH}}_{2} \mathrm{O}\right), 67.1\left(\mathrm{~s}, \mathrm{CO}_{2} \underline{\mathrm{CH}}_{2}\right), 85.3$ (dd, $\left.{ }^{1} J_{\mathrm{CF} / \mathrm{P}}=195.6,158.4, \mathrm{PCHF}\right), 119.8\left(\mathrm{~s}, \mathrm{CO}_{2} \mathrm{CH}_{2}-\mathrm{CH}=\mathrm{CH}_{2}\right), 131.2\left(\mathrm{~s}, \mathrm{CO}_{2} \mathrm{CH}_{2}-\underline{\mathrm{CH}}=\mathrm{CH}_{2}\right), 164.9\left(\mathrm{~d},{ }^{2} J_{\mathrm{CF}}\right.$ or $\left.\mathrm{CP}=21.9, \mathrm{PCHF}-\underline{C O}_{2}\right) . / \mathbf{m} / \mathbf{z}(\mathrm{EI}) 255\left(\mathrm{M}^{+}+1,11 \%\right), 227$ (7), 197 (37), 170 (50).

Diethyl isobutyl 1-fluoromethylphosphonocarboxylate (4g) : $\mathrm{C}_{10} \mathrm{H}_{20} \mathrm{O}_{5} \mathrm{PF}(\mathrm{M}=270)$

NMR ${ }^{31} \mathbf{P}\left(\mathrm{CDCl}_{3}\right)+10.4\left(\mathrm{~d},{ }^{2} J_{\mathrm{PF}}=72.3\right) . /{ }^{\mathbf{1}} \mathbf{H}\left(\mathrm{CDCl}_{3}\right) 0.94\left(\mathrm{~d}, 6 \mathrm{H},{ }^{3} J_{\mathrm{HH}}=6.7, \mathrm{CH}\left(\mathrm{CH}_{3}\right)_{2}\right), 1.34(\mathrm{tm}, 6 \mathrm{H}$, $\left.{ }^{3} J_{\mathrm{HH}}=7.0, \mathrm{C}_{3} \mathrm{CH}_{2} \mathrm{O}\right), 1.98\left(\mathrm{~m}, 1 \mathrm{H},{ }^{3} J_{\mathrm{HH}}=6.7, \mathrm{C} \underline{\mathrm{H}}\left(\mathrm{CH}_{3}\right)_{2}\right), 4.03\left(2 \mathrm{~d}, 2 \mathrm{H},{ }^{3} J_{\mathrm{HH}}=6.7, \Delta J=3.5, \mathrm{CO}_{2} \mathrm{C}_{2}\right), 4.23$ $\left(\mathrm{dqd}, 4 \mathrm{H},{ }^{3} J_{\mathrm{HP}}=8.2,{ }^{3} J_{\mathrm{HH}}=7.0,{ }^{5} J_{\mathrm{HF}}=3.0, \mathrm{CH}_{3} \mathrm{CH}_{2} \mathrm{O}\right), 5.20\left(\mathrm{dd}, 1 \mathrm{H},{ }^{2} J_{\mathrm{HF} / \mathrm{P}}=47.0,12.4, \mathrm{PC} \underline{\mathrm{HF}}\right) . /{ }^{13} \mathrm{C}\left(\mathrm{CDCl}_{3}\right)$ $16.5\left(\mathrm{~d},{ }^{3} J_{\mathrm{CP}}=5.5, \underline{\mathrm{CH}}_{3} \mathrm{CH}_{2} \mathrm{O}\right), 19.1\left(\mathrm{~s}, \mathrm{CH}\left(\underline{\mathrm{CH}}_{3}\right)_{2}\right), 27.9\left(\mathrm{~s}, \underline{\mathrm{CH}}\left(\mathrm{CH}_{3}\right)_{2}\right), 64.3\left(\mathrm{t},{ }^{2} J_{\mathrm{CP}}=5.7, \mathrm{CH}_{3} \underline{\mathrm{CH}}{ }_{2} \mathrm{O}\right), 72.3$ 
(s, $\left.\mathrm{CO}_{2} \underline{\mathrm{CH}_{2}}\right), 85.1\left(\mathrm{dd},{ }^{1} J_{\mathrm{CF} / \mathrm{P}}=195.4,158.6, \mathrm{P} \underline{\mathrm{HF}}\right), 165.1\left(\mathrm{~d},{ }^{2} J_{\mathrm{CF}}\right.$ or $\left.\mathrm{CP}=22.0, \mathrm{PCHF}-\underline{\mathrm{CO}}_{2}\right) . / \mathbf{~ m} / \mathbf{z}(\mathrm{EI}) 271$ $\left(\mathrm{M}^{+}+1,4 \%\right), 314(30), 197(23), 186(56)$.

Diethyl neopentyl 1-fluoromethylphosphonocarboxylate (4h) : $\mathrm{C}_{11} \mathrm{H}_{22} \mathrm{O}_{5} \mathrm{PF}(\mathrm{M}=284)$

NMR ${ }^{31} \mathbf{P}\left(\mathrm{CDCl}_{3}\right)+10.2\left(\mathrm{~d},{ }^{2} J_{\mathrm{PF}}=72.7\right)$. / ${ }^{\mathbf{1}} \mathbf{H}\left(\mathrm{CDCl}_{3}\right) 1.25\left(\mathrm{~s}, 9 \mathrm{H}, \mathrm{C}\left(\mathrm{C}_{3}\right)_{3}\right), 1.33\left(\mathrm{tm}, 6 \mathrm{H},{ }^{3} J_{\mathrm{HH}}=7.1\right.$, $\mathrm{CH}_{3} \mathrm{CH}_{2} \mathrm{O}$ ), 3.88 (A part of $\mathrm{AB}$ system, $1 \mathrm{H},{ }^{2} J_{\mathrm{HHgem}}=10.5, \mathrm{CO}_{2} \mathrm{CH}_{\mathrm{A}} \mathrm{H}_{\mathrm{B}}$ ), 3.99 (B part of AB system, $1 \mathrm{H}$, $\left.{ }^{2} J_{\mathrm{HHgem}}=10.5, \mathrm{CO}_{2} \mathrm{CH}_{\mathrm{A}} \underline{\mathrm{H}}_{\mathrm{B}}\right), 4.22\left(\mathrm{dqd}, 4 \mathrm{H},{ }^{3} J_{\mathrm{HP}}=8.3,{ }^{3} J_{\mathrm{HH}}=7.1,{ }^{5} J_{\mathrm{HF}}=2.6, \mathrm{CH}_{3} \underline{\mathrm{H}}_{2} \mathrm{O}\right), 5.20(\mathrm{dd}, 1 \mathrm{H}$, $\left.{ }^{2} J_{\mathrm{HF} / \mathrm{P}}=46.9,12.4, \mathrm{PC} \underline{\mathrm{HF}}\right) . /{ }^{13} \mathrm{C}\left(\mathrm{CDCl}_{3}\right) 16.7\left(\mathrm{~d},{ }^{3} J_{\mathrm{CP}}=5.7, \underline{\mathrm{CH}}_{3} \mathrm{CH}_{2} \mathrm{O}\right), 26.6\left(\mathrm{~s}, \mathrm{C}\left(\underline{\mathrm{CH}_{3}}\right)_{3}\right), 31.9$ (s, $\left.\underline{\mathrm{C}}\left(\mathrm{CH}_{3}\right)_{3}\right), 64.5\left(\mathrm{t},{ }^{2} J_{\mathrm{CP}} /{ }^{4} J_{\mathrm{CF}}=5.4, \mathrm{CH}_{3} \underline{\mathrm{CH}}_{2} \mathrm{O}\right), 75.7\left(\mathrm{~s}, \mathrm{CO}_{2} \underline{\mathrm{CH}_{2}}\right), 85.2\left(\mathrm{dd},{ }^{1} J_{\mathrm{CF} / \mathrm{P}}=195.3,158.6, \mathrm{P} \underline{\mathrm{HF}}\right)$, $165.2\left(\mathrm{~d},{ }^{2} J_{\mathrm{CF}}\right.$ or $\left.\mathrm{CP}=22.0, \mathrm{PCHF}-\underline{C O}_{2}\right) . / \mathbf{m} / \mathbf{z}(\mathrm{EI}) 285\left(\mathrm{M}^{+}+1,1 \%\right), 269$ (13), 241 (11), 228 (44).

Diethyl isopropyl 1-fluoromethylphosphonocarboxylate (4i) : $\mathrm{C}_{9} \mathrm{H}_{18} \mathrm{O}_{5} \mathrm{PF}(\mathrm{M}=256)$

NMR ${ }^{31} \mathbf{P}\left(\mathrm{CDCl}_{3}\right)+9.7\left(\mathrm{~d},{ }^{2} J_{\mathrm{PF}}=72.4\right) . /{ }^{1} \mathbf{H}\left(\mathrm{CDCl}_{3}\right) 1.28\left(\mathrm{dd}, 6 \mathrm{H},{ }^{3} J_{\mathrm{HH}}=6.2,1.0, \mathrm{CH}\left(\mathrm{CH}_{3}\right)_{2}\right), 1.33(\mathrm{tm}, 6 \mathrm{H}$,

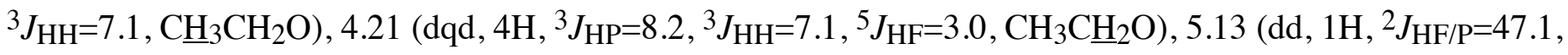
12.4, PCㅌF), $5.16\left(\mathrm{p}, 1 \mathrm{H},{ }^{3} J_{\mathrm{HH}}=6.2, \mathrm{C} \underline{\mathrm{H}}\left(\mathrm{CH}_{3}\right)_{2}\right) . /{ }^{13} \mathrm{C}\left(\mathrm{CDCl}_{3}\right) 16.3\left(\mathrm{~d},{ }^{3} J_{\mathrm{CP}}=5.7, \underline{\mathrm{CH}}_{3} \mathrm{CH}_{2} \mathrm{O}\right), 21.5(\mathrm{~d}$, $\left.J=7.8, \mathrm{CH}\left(\underline{\mathrm{CH}}_{3}\right)_{2}\right), 64.0\left(\mathrm{dd},{ }^{2} J_{\mathrm{CP}} /{ }^{4} J_{\mathrm{CF}}=6.1,2.5, \mathrm{CH}_{3} \underline{\mathrm{CH}}_{2} \mathrm{O}\right), 70.4\left(\mathrm{~s}, \underline{\mathrm{CH}}\left(\mathrm{CH}_{3}\right)_{2}\right), 84.9\left(\mathrm{dd},{ }^{1} J_{\mathrm{CF} / \mathrm{P}}=195.2\right.$,

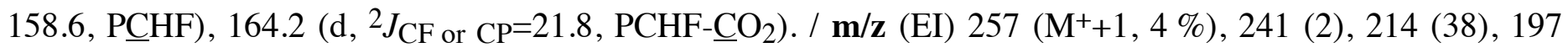
(71).

Diethyl 1,3-dichloroisopropyl 1-fluoromethylphosphonocarboxylate $(\mathbf{4 j}): \mathrm{C}_{9} \mathrm{H}_{16} \mathrm{O}_{5} \mathrm{PFCl}_{2}(\mathrm{M}=325)$

NMR ${ }^{31} \mathbf{P}\left(\mathrm{CDCl}_{3}\right)+10.5\left(\mathrm{~d},{ }^{2} J_{\mathrm{PF}}=72.5\right)$. / ${ }^{1} \mathbf{H}\left(\mathrm{CDCl}_{3}\right) 1.37\left(\mathrm{t}, 6 \mathrm{H},{ }^{3} J_{\mathrm{HH}}=7.1, \mathrm{C}_{3} \mathrm{CH}_{2} \mathrm{O}\right), 3.78(\mathrm{~d}, 4 \mathrm{H}$, $\left.{ }^{3} J_{\mathrm{HH}}=5.3, \mathrm{CH}\left(\mathrm{CH}_{2} \mathrm{Cl}\right)_{2}\right), 4.26\left(\mathrm{dqd}, 4 \mathrm{H},{ }^{3} J_{\mathrm{HP}}=8.3,{ }^{3} J_{\mathrm{HH}}=7.0,{ }^{5} J_{\mathrm{HF}}=1.6, \mathrm{CH}_{3} \underline{\mathrm{H}}_{2} \mathrm{O}\right), 5.27(\mathrm{dd}, 1 \mathrm{H}$,

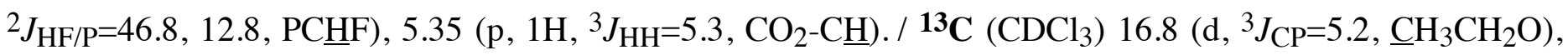
$42.3\left(2 \mathrm{~s}, \Delta J=13.9, \mathrm{CH}\left(\underline{\mathrm{CH}}{ }_{2} \mathrm{Cl}\right)_{2}\right), 64.9\left(\mathrm{t},{ }^{2} J_{\mathrm{CP}}=6.3, \mathrm{CH}_{3} \underline{\mathrm{CH}}_{2} \mathrm{O}\right), 74.1\left(\mathrm{~s}, \mathrm{CO}_{2}-\underline{\mathrm{CH}}\right), 85.2\left(\mathrm{dd},{ }^{1} J_{\mathrm{CF} / \mathrm{P}}=196.4\right.$, 158.5, PCHF), 164.5 (d, ${ }^{2} J_{\mathrm{CF}}$ or $\left.\mathrm{CP}=22.6, \mathrm{PCHF}-\underline{C O}_{2}\right) . / \mathbf{m} / \mathbf{z}(\mathrm{EI}) 325\left(\mathrm{M}^{+}+1,2^{35} \mathrm{Cl}, 6 \%\right), 297\left(2^{35} \mathrm{Cl}, 8\right), 261$ $\left(1^{35} \mathrm{Cl}, 20\right), 197(100)$.

Diethyl vinyll-fluoromethylphosphonocarboxylate (4k) : $\mathrm{C}_{8} \mathrm{H}_{14} \mathrm{O}_{5} \mathrm{PF}(\mathrm{M}=240)$

NMR ${ }^{31} \mathbf{P}\left(\mathrm{CDCl}_{3}\right)+10.2\left(\mathrm{~d},{ }^{2} \mathrm{~J}_{\mathrm{PF}}=71.9\right)$. / ${ }^{\mathbf{1}} \mathbf{H}\left(\mathrm{CDCl}_{3}\right) 1.35\left(\mathrm{tm}, 6 \mathrm{H},{ }^{3} \mathrm{~J}_{\mathrm{HH}}=7.1, \mathrm{CH}_{3} \mathrm{CH}_{2} \mathrm{O}\right), 4.25(\mathrm{dqd}, 4 \mathrm{H}$, $\left.{ }^{3} J_{\mathrm{HP}}=8.1,{ }^{3} J_{\mathrm{HH}}=7.1,{ }^{5} J_{\mathrm{HF}}=3.8, \mathrm{CH}_{3} \mathrm{CH}_{2} \mathrm{O}\right), 4.74\left(\mathrm{dd}, 1 \mathrm{H},{ }^{3} J_{\mathrm{HHcis}}=6.2,{ }^{2} J_{\mathrm{HHgem}}=2.1, \mathrm{CO}_{2} \mathrm{CH}=\mathrm{CH}_{\mathrm{A}} \mathrm{H}_{\mathrm{B}}\right), 5.04$ $\left(\mathrm{dd}, 1 \mathrm{H},{ }^{3} J_{\mathrm{HHtrans}}=13.8,{ }^{2} J_{\mathrm{HHgem}}=2.1, \mathrm{CO}_{2} \mathrm{CH}=\mathrm{CH}_{\mathrm{A}} \underline{\mathrm{H}}_{\mathrm{B}}\right), 5.28\left(\mathrm{dd}, 1 \mathrm{H},{ }^{2} J_{\mathrm{HF} / \mathrm{P}}=46.7,13.0, \mathrm{PC} \underline{\mathrm{HF}}\right), 7.29(\mathrm{dd}$, $\left.1 \mathrm{H},{ }^{3} J_{\mathrm{HHtrans}}=13.8,{ }^{3} J_{\mathrm{HHcis}}=6.2, \mathrm{CO}_{2}-\mathrm{CH}=\right) . /{ }^{13} \mathrm{C}\left(\mathrm{CDCl}_{3}\right) 16.7\left(\mathrm{~d},{ }^{3} J_{\mathrm{CP}}=5.2, \underline{\mathrm{CH}}_{3} \mathrm{CH}_{2} \mathrm{O}\right), 64.9\left(\mathrm{~d},{ }^{2} J_{\mathrm{CP}}=6.6\right.$, $\left.\mathrm{CH}_{3} \underline{\mathrm{CH}}_{2} \mathrm{O}\right), 85.0\left(\mathrm{~d},{ }^{1} J_{\mathrm{CF} / \mathrm{P}}=196.3,157.8, \mathrm{P} \underline{\mathrm{CHF}}\right), 100.5\left(\mathrm{~s}, \mathrm{CO}_{2}-\mathrm{CH}=\underline{\mathrm{CH}}_{2}\right), 140.7\left(\mathrm{~s}, \mathrm{CO}_{2}-\underline{\mathrm{CH}}=\mathrm{CH}_{2}\right), 162.6$ $\left(\mathrm{d},{ }^{2} J_{\mathrm{CF}}\right.$ or $\left.\mathrm{CP}=22.7, \mathrm{PCHF}-\underline{\mathrm{CO}}_{2}\right) . / \mathbf{~ m} / \mathbf{z}(\mathrm{EI}) 241\left(\mathrm{M}^{+}+1,2 \%\right), 213$ (1), 197 (100), 169 (39).

Diethyl phenyl 1-fluoromethylphosphonocarboxylate (41) : $\mathrm{C}_{12} \mathrm{H}_{16} \mathrm{O}_{5} \mathrm{PF}(\mathrm{M}=290)$

NMR ${ }^{31} \mathbf{P}\left(\mathrm{CDCl}_{3}\right)+8.9\left(\mathrm{~d},{ }^{2} J_{\mathrm{PF}}=71.9\right)$. / ${ }^{\mathbf{1}} \mathbf{H}\left(\mathrm{CDCl}_{3}\right) 1.34\left(\mathrm{t}, 6 \mathrm{H},{ }^{3} \mathrm{~J}_{\mathrm{HH}}=7.1, \mathrm{C}_{3} \mathrm{CH}_{2} \mathrm{O}\right), 4.27(\mathrm{dqd}, 4 \mathrm{H}$,

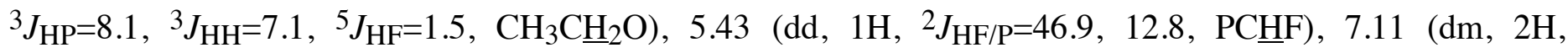
${ }^{3} J_{\mathrm{HH}}=7.4, \underline{\mathrm{H}}_{\text {ortho }}$ de $\left.\mathrm{C}_{6} \mathrm{H}_{5}\right), 7.22\left(\mathrm{tt}, 1 \mathrm{H},{ }^{3} J_{\mathrm{HH}}=7.4,{ }^{4} J_{\mathrm{HH}}=1.3, \underline{\mathrm{H}}_{\text {para }}\right.$ de $\left.\mathrm{C}_{6} \mathrm{H}_{5}\right), 7.36\left(\mathrm{tm}, 2 \mathrm{H},{ }^{3} J_{\mathrm{HH}}=7.4, \underline{\mathrm{H}}_{\text {meta }}\right.$ de $\left.\mathrm{C}_{6} \mathrm{H}_{5}\right) . /{ }^{13} \mathrm{C}\left(\mathrm{CDCl}_{3}\right) 16.8\left(\mathrm{~d},{ }^{3} J_{\mathrm{CP}}=5.2 \quad \mathrm{CH}_{3} \mathrm{CH}_{2} \mathrm{O}\right), 65.0\left(\mathrm{~d},{ }^{2} J_{\mathrm{CP}}=6.4, \mathrm{CH}_{3} \underline{C H}_{2} \mathrm{O}\right), 85.5$ (dd, ${ }^{1} J_{\mathrm{CF} / \mathrm{P}}=196.8,158.3, \mathrm{P} \underline{\mathrm{HF}}$ ), 121.6 (s, $\underline{\mathrm{C}}_{\text {ortho }}$ de $\mathrm{C}_{6} \mathrm{H}_{5}$ ), 127.1 (s, $\underline{\mathrm{C}}_{\text {para }}$ de $\mathrm{C}_{6} \mathrm{H}_{5}$ ), 130.1 (s, $\underline{\mathrm{C}}_{\text {meta }}$ de $\left.\mathrm{C}_{6} \mathrm{H}_{5}\right)$, 
150.3 (s, $\underline{\mathrm{C}}_{\mathrm{ipso}}$ de $\left.\mathrm{C}_{6} \mathrm{H}_{5}\right), 164.1\left(\mathrm{~d},{ }^{2} J_{\mathrm{CF}}\right.$ or $\left.\mathrm{CP}=22.7, \mathrm{PCHF}^{-\mathrm{CO}_{2}}\right) . /$ m/z $(\mathrm{EI}) 290\left(\mathrm{M}^{+}, 17 \%\right), 245$ (3), 217 (10), 197 (100).

1-fluoromethyl(diethylphosphono)carboxylic acid (7) : To a solution of $n$-BuLi $(8 \mathrm{~mL}$ of a $1.6 \mathrm{M}$ solution in hexane, $13 \mathrm{mmol})$ in THF $(15 \mathrm{~mL})$ at $-78^{\circ} \mathrm{C}$ is slowly added a mixture of TMSCl $(0.66 \mathrm{~g}$; $6.1 \mathrm{mmol})$ and diethyl 1,1-dibromo-1-fluoromethylphosphonate $(2 \mathrm{~g}, 6.1 \mathrm{mmol})$ in THF (15 mL) by means of a dropping funnel maintaining the temperature at $-78^{\circ} \mathrm{C}$. Stirring was continued for $10 \mathrm{~min}$ at this temperature before a source of dry $\mathrm{CO}_{2}$ gas was introduced into the rapidly stirred reaction mixture, an exothermic reaction takes place and the reaction is allowed to warm to $-60^{\circ} \mathrm{C}$. $\mathrm{CO}_{2}$ addition is continued for a further $15 \mathrm{~min}$ at $60^{\circ} \mathrm{C}$. The reaction mixture is slowly allowed to warm to $20^{\circ} \mathrm{C}$ and stirred for $10 \mathrm{~min}$ at this temperature. The reaction mixture is then poured into a rapidly stirred mixture of dichloromethane $(50 \mathrm{~mL})$ and $3 \mathrm{M} \mathrm{HCl}$ $(50 \mathrm{~mL})$ and ice $(10 \mathrm{~g})$. This mixture is stirred for a further $10 \mathrm{~min}$ before the phases are separated and the aqueous phase is extracted with dichloromethane $(3 \times 20 \mathrm{~mL})$. The combined organic phases are dried over $\mathrm{MgSO}_{4}$ and then filtered and evaporated to yield the title compound 7 as a white crystalline solid (mp 70$74^{\circ} \mathrm{C}$ ).

NMR 31P $\left(\mathrm{CDCl}_{3}\right)+11.03\left(\mathrm{~d},{ }^{2} J_{\mathrm{PF}}=72.6\right)$. / ${ }^{\mathbf{1}} \mathbf{H}\left(\mathrm{CDCl}_{3}\right)$ 1.24-1.50 (m, 6H, $\left.\mathrm{CH}_{3} \mathrm{CH}_{2} \mathrm{O}\right), 4.12-4.36(\mathrm{~m}, 4 \mathrm{H}$,

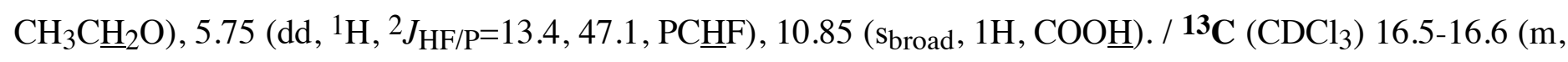
$\left.\underline{\mathrm{CH}}_{3} \mathrm{CH}_{2} \mathrm{O}\right), 65.4-65.7\left(\mathrm{~m}, \mathrm{CH}_{3} \underline{\mathrm{CH}}_{2} \mathrm{O}\right), 84.6\left(\mathrm{dd},{ }^{1} J_{\mathrm{CF} / \mathrm{P}}=195.1,159.9\right.$, P $\left.\underline{\mathrm{CHF}}\right), 166.4\left(\mathrm{~d},{ }^{2} \mathrm{~J}_{\mathrm{CF}}\right.$ or $\mathrm{CP}=19$, $\mathrm{COOH}) . / \mathbf{m} / \mathbf{z}(\mathrm{EI}) 214\left(\mathrm{M}^{+}\right)$.

\section{ACKNOWLEDGEMENTS}

The authors are grateful to Dr. J. P. Senet and Dr. G. Sennyey (ISOCHEM Groupe SNPE, 91710 Vert-LePetit, France) for the generous gift of chloroformates. We thank the CNRS for financial support (to R.W. and J.C.). We wish to thank Mr. M. Levard (URA CNRS 1307, Ecole Polytechnique) for the mass spectra analyses.

\section{REFERENCES}

1. Welch, J.T. Tetrahedron $\mathbf{1 9 8 7}, 43,3123$

2. (a) Thenappan, A.; Burton, D.J. J. Org. Chem. 1990, 55, 4639;

(b) Camps, F.; Coll, J.; Fabrias, G.; Guerrero, A. Tetrahedron 1984, 40, 2871; McCarthy, J.R.; Mathews, D.P.; Edwards, M.L. Stemerick, D.M.; Jarvi, E.T. Tetrahedron Lett. 1990, 38, 5449; Patrick, T.B.; Nadjii, S. J. Fluorine Chem. 1990, 49, 147.

3. McDonald, I.A.; Lacoste, J.M.; Bey, P.; Wagner, J.; Zreika, M.; Palfreyman, M.G. J. Am. Chem. Soc. 1984, 106, 3354; McCarthy, J.R.; Jarvi, E.T.; Mathews, D.P.; Edwards, M.L.; Prakash, N.J.; Bowlin, T.L.; Mehdi, S.; Sunkara, P.S.; Bey, P. J. Am. Chem. Soc. 1989, 111, 1127. 
4. Ziegler, E.; Wittman, H. Monatsh. Chem. 1986, 117, 653.

5. Boguslavskaya, L.S.; Panteleeva, I.Y.; Morozova, T.V.; Kartashov, A.V.; Chuvatkin, N.N. Russian Chem. Revs. 1990, 59, 906.

6. (a) Patois, C.; Savignac, P. J. Chem. Soc., Chem.Comm. 1993, 1711;

(b) Waschbüsch, R.; Carran, J.; Savignac, P. Tetrahedron 1996, 52, 14199;

(c) Waschbüsch, R.; Carran, J.; Savignac, P. J. Chem. Soc, Perkin Trans. I 1996, in press.

7. (a) Etemad-Moghadam, G.; Seyden-Penne, J. Bull. Soc. Chim. Fr. 1985, 3, 448;

(b) Machleidt, H.; Wessendorf, R. Justus Liebigs Ann. Chem. 1964, 674, 1; Greil, W.; Machleidt, H. Justus Liebigs Ann. Chem. 1966, 693, 134;

(c) Fokin, A. V.; Zimin, V.I.; Studnev, Yu. N.; Rapkin, A.I. Zh. Org. Khim. 1971, 7, 249 (Engl. Transl. 1971, 7, 241; C.A.74 124736s);

(d) Davis, F.A.; Han, W.; Murphy, C.K. J. Org. Chem. 1995, 15, 4730; Davis, F.A.; Han, W. Tetrahedron Lett. 1991, 32, 1631;

(e) Grell, W.; Machleidt, H. Justus Liebigs Ann. Chem. 1966, 693, 134;

(f) Thenappan, A.; Burton, D.J. J. Org. Chem. 1991, 56, 273;

(g) Patois, C.; Savignac, P. Phosphorus, Sulfur, Silicon, Relat. Elem. 1993, 77, 163;

(h) Massoudi, H.H.; Cantacuzene, D.; Wakselman, C; Bouthier de la Tour, C. Synthesis 1983, 12, 1010.

8. (a) Thenappan, A.; Burton, D.J. J. Org. Chem. 1990, 55, 2311;

(b) Elkik, E.; Imbeaux, M. Synthesis 1989, 11, 861;

(c) Tsai, H.J.; Thenappan, A.; Burton, D.J.J. Org. Chem. 1994, 59, 7085;

(d) Tsai, H.J.; Thenappan, A.; Burton, D.J. Phosphorus, Sulfur, Silicon, Relat. Elem. 1995, 105, 1-4, 205.

9. Coutrot, P.; Grison, C.; Sauvêtre, R. J. Organomet. Chem. 1987, 332, 1.

10. For a review of fluorinated ylids and related compounds see Burton, D.J.; Yang, Z.-Y.; Qiu, W. Chem. Rev. 1996, 96, 1641.

11. For reviews on the selective fluorination of organic molecules see Purrington, S.T.; Kagan, B.S.; Patrick, T.B. Chem. Rev. 1986, 86, 997; Wilkinson, J.A. Chem. Rev. 1992, 92, 505; Lal, S. Chem. Rev. 1996, 96, 1737. 